\title{
Nuevas combinaciones en los géneros sudamericanos Lepidaploa y Lessingianthus (Vernonieae, Asteraceae)
}

\section{New combinations in the South American genera Lepidaploa and Lessingianthus (Vernonieae, Asteraceae)}

\author{
María Betiana Angulo*, Álvaro José Vega \& Massimiliano Dematteis \\ Instituto de Botánica del Nordeste (UNNE-CONICET), Casilla de Correo 209, 3400 Corrientes, Argentina. \\ *angulobetiana@gmail.com
}

\begin{abstract}
RESUMEN
Los géneros Lepidaploa (Cass.) Cass. y Lessingianthus H. Rob. (Vernonieae, Asteraceae) fueron establecidos para separar a las especies inicialmente ubicadas en Vernonia Schreb. sect. Lepidaploa (Cass.) DC. subsect. Axilliflorae Benth. \& Hook. y Vernonia sect. Lepidaploa subsect. Macrocephalae Benth. \& Hook., respectivamente. Tal como se delimitan actualmente, Lepidaploa está compuesto por alrededor de 150 especies ampliamente distribuidas en Sudamérica, mientras que Lessingianthus presenta 120 especies con una gran concentración en el sudeste de Brasil. Se estima que gran cantidad de especies todavía incluidas en Vernonia no han sido estudiadas apropiadamente y en consecuencia su posición y estatus taxonómicos permanecen inciertos. En este trabajo se analizó la morfología de los granos de polen y de algunos microcaracteres florales para aportar datos que permitieron transferir seis taxones de Vernonia al género Lessingianthus y otras dos especies del mismo género a Lepidaploa.
\end{abstract}

Palabras clave: Vernonia, Lepidaploinae, Vernoniinae, sistemática.

\begin{abstract}
The genera Lepidaploa (Cass.) Cass. and Lessingianthus H. Rob. (Vernonieae, Asteraceae) were established to separate taxa initially placed in Vernonia Schreb. sect. Lepidaploa (Cass.) DC. subsect. Axilliflorae Benth. \& Hook. and Vernonia sect. Lepidaploa subsect. Macrocephalae Benth. \& Hook., respectively. As presently delimited, Lepidaploa is comprised of about 150 species widely distributed in South America, while Lessingianthus presents 120 species having a concentration in southeastern Brazil. It is estimated that a large amount of several species still included in Vernonia have not been examined and consequently their taxonomic position remains uncertain. We analyzed pollen morphology and floral microcharacters of several Vernonia species, which allowed the transfer of six taxa previously placed in this genus to Lessingianthus and other two species of the Vernonia to Lepidaploa.
\end{abstract}

KeYworDs: Vernonia, Lepidaploinae, Vernoniinae, systematics.

\section{INTRODUCCIÓN}

La tribu Vernonieae Cass. constituye uno de los grupos más grandes de Compuestas (Asteraceae), con alrededor de 120 géneros y más de 1.700 especies distribuidas en las regiones tropicales de Asia, África y América (Angulo \& Dematteis 2012). De estos géneros, 48 son monotípicos y otros 30 tienen sólo dos especies, mientras que la mayoría de las especies se encuentran en unos pocos géneros de la tribu (Robinson 2007). Los géneros americanos se hallan agrupados actualmente en 10 subtribus, que se distinguen principalmente por el tipo de polen, el número de flores, el tipo de receptáculo, el número cromosómico y la presencia de metabolitos secundarios como lactonas sesquiterpénicas (Keeley \& Robinson 2009). En Sudamérica, las Vernonieae están representadas por alrededor de 350-360 especies que habitan principalmente en el norte de Argentina, Bolivia, Brasil y Paraguay (Robinson 2007).

Las Vernonieae son consideradas uno de los grupos más complejos de la familia Asteraceae desde el punto de vista biológico y taxonómico. Las mayores discusiones han girado en torno a la delimitación de Vernonia Schreb., el 
género más grande de la tribu, sobre el cual aún no existe un acuerdo satisfactorio (Jones 1979). La gran variación en hábito y morfología de las especies, llevó a diferentes autores a adoptar distintos criterios en la delimitación taxonómica a nivel genérico o infragenérico. Robinson (1999) circunscribe Vernonia principalmente a Norteamérica y segrega casi todas las especies sudamericanas a nuevos géneros.

Tal como se delimita actualmente la tribu, dos de los grupos más grandes son los géneros Lessingianthus $\mathrm{H}$. Rob. [= Vernonia sect. Lepidaploa (Cass.) DC. subsect. Macrocephalae Benth. \& Hook.] y Lepidaloa (Cass.) Cass. [= Vernonia sect. Lepidaploa (Cass.) DC. subsect. Axilliflorae Benth. \& Hook.]. El género Lepidaploa está compuesto por alrededor de 150 especies ampliamente distribuidas en Sudamérica, mientras que Lessingianthus presenta 120 especies con una gran concentración en el sudeste de Brasil. Este último comprende plantas con capítulos grandes, reunidos en inflorescencias cimosas, anteras con apéndice apical sin glándulas, estilos sin nódulo basal y cristales de la pared del fruto cúbicos (Dematteis 2006, Robinson 2007). Por su parte, las especies de Lepidaploa se caracterizan por la presencia de capítulos pequeños, dispuestos en inflorescencias cimosas, anteras con apéndice apical raramente glanduloso, estilos con nódulo basal y cristales de la pared del fruto prismáticos (Robinson 1990, 2007).

Uno de los caracteres que permite distinguir estos dos grupos entre sí es la morfología del polen. Lessingianthus se caracteriza por presentar polen tipo "B", los cuales son tricolporados, equinolofados, con el tectum discontinuo con lagunas ecuatoriales y sin lagunas polares (Dematteis \& Pire 2008, Angulo \& Dematteis 2010). Los granos de polen de Lepidaploa pueden ser de tipo "C", "D" o "G". Estos tres tipos de granos también son tricolporados, equinolofados y con el tectum discontinuo, pero se diferencian en que el " $\mathrm{C}$ " tiene lagunas polares y carece de lagunas ecuatoriales, mientras que el "D" no presenta lagunas polares ni ecuatoriales y el lofo no separa completamente la laguna abporal de la poral. Finalmente el tipo "G" carece de laguna polar y colpos divididos (Robinson 1992, Dematteis \& Pire 2008).

Desde la descripción de los géneros Lepidaploa (Robinson 1990) y Lessingianthus (Robinson 1988) no se han producido discusiones con respecto a los límites de ambos grupos. Las únicas novedades han sido la descripción de nuevas especies o combinaciones y la resolución de algunos problemas nomenclaturales puntuales (Dematteis 2006, 2008, Borges \& Dematteis 2008, Dematteis \& Angulo 2010, 2012). Sin embargo, algunas especies todavía incluidas en Vernonia no han sido estudiadas apropiadamente y en consecuencia su posición y estatus taxonómicos permanecen inciertos. El objetivo de este trabajo fue transferir seis especies de Vernonia a Lessingianthus y otras dos entidades del mismo género a Lepidaploa sobre la base de su morfología polínica y de sus microcaracteres florales.

\section{MATERIALES Y MÉTODOS}

El estudio se realizó mediante el análisis de caracteres morfológicos de ejemplares depositados en los herbarios BA, BAF, C, CORD, CTES, FCQ, G, K, L. LIL, LP, LPB, M, MA, MBM, MCNS, P, S, SI, SP, US, USZ y W.

Los granos de polen fueron obtenidos a partir de 1 ó 2 botones florales provenientes de ejemplares de herbario. Todas las muestras fueron sometidas a la técnica de acetólisis sugerida por Erdtman (1966). Para la observación con el microscopio óptico, los granos de polen fueron montados en gelatina glicerinada y luego analizados con microscopio Zeiss Axioplan. Los preparados permanentes fueron depositados en el laboratorio de palinología de la Universidad Nacional del Nordeste (PAL-CTES). La terminología utilizada para describir la morfología del polen es la sugerida por Erdtman (1966), Punt et al. (1994) y Keeley \& Jones (1979).

Los microcaracteres fueron analizados a partir de flores obtenidas de ejemplares de herbario. Las mismas fueron hidratadas en agua hirviendo a la cual se le agregó una gotita de detergente. Posteriormente se procedió a la disección de las flores bajo microscopio estereoscópico y se montaron en solución de Hoyer (Anderson 1954).

\section{RESULTADOS}

\section{Lepidaploa}

Dos especies pertenecientes al género Vernonia, $V$. novarae Cabrera y $V$. setososquamosa Hieron., presentaron caracteres distintivos del género Lepidaploa, tales como la morfología de los granos de polen y algunos microcaracteres morfológicos. Ello hizo necesario la transferencia de ambas especies a este último género.

Vernonia novarae mostró granos de polen tipo " $D$ " (Fig. $1 \mathrm{~A}-\mathrm{C})$, los cuales son tricolporados, equinolofados. Los colpos son largos, con ápices visibles en vista polar, el colpo interrumpe el lofo que separa la laguna poral de las abporales. El tamaño del polen: $\mathrm{P}=47,3 \mu \mathrm{m}, \mathrm{E}=49,2 \mu \mathrm{m}$. El grosor de la exina, excluyendo las espinas, osciló entre 4,0-5,4 $\mu \mathrm{m}$. Lagunas de 8,6-12,2 $\mu \mathrm{m}$. diám., dispuestas en un modelo regular, sin laguna polar. Espinas de 4,0-5,4 $\mu \mathrm{m}$ long. En cuanto a los microcaracteres florales, esta entidad presentó un nódulo en la base del estilo (Fig. 2 A-B) y los cristales prismáticos en la pared del fruto (Fig. 2 C-D).

Vernonia setososquamosa presentó polen tipo "C" (Fig. 1 D-F), los cuales son tricolporados, equinolofados. Los colpos son largos. $\mathrm{P}=37,8 \mu \mathrm{m}, \mathrm{E}=38,0 \mu \mathrm{m}$. El grosor de la exina, excluyendo las espinas, osciló entre 4,5-5,5 $\mu \mathrm{m}$. Lagunas de 7-9 $\mu \mathrm{m}$. diám., con contorno más o menos regular, laguna polar presente. Espinas de 1,3-2,04 $\mu \mathrm{m}$ long., agudas en el ápice. Esta especie también presentó nódulo basal estilar y cristales prismáticos en la pared fruto. 
Lepidaploa novarae (Cabrera) A.J. Vega \& Dematt. comb. nov. Basónimo: Vernonia novarae Cabrera, Hickenia 2: 255. 1997. TIPO: "Argentina. Prov. Salta, Dpto. Santa Victoria: Parque Nacional Baritú, faldeos al E de la sierra del Porongal, entre Quebrada La Gateada y Quebrada Seca, 1400-1800 m s.m., 20.IX.1990, Novara 9993” (holotipo, MCNS!; isotipos, M!, SI!).
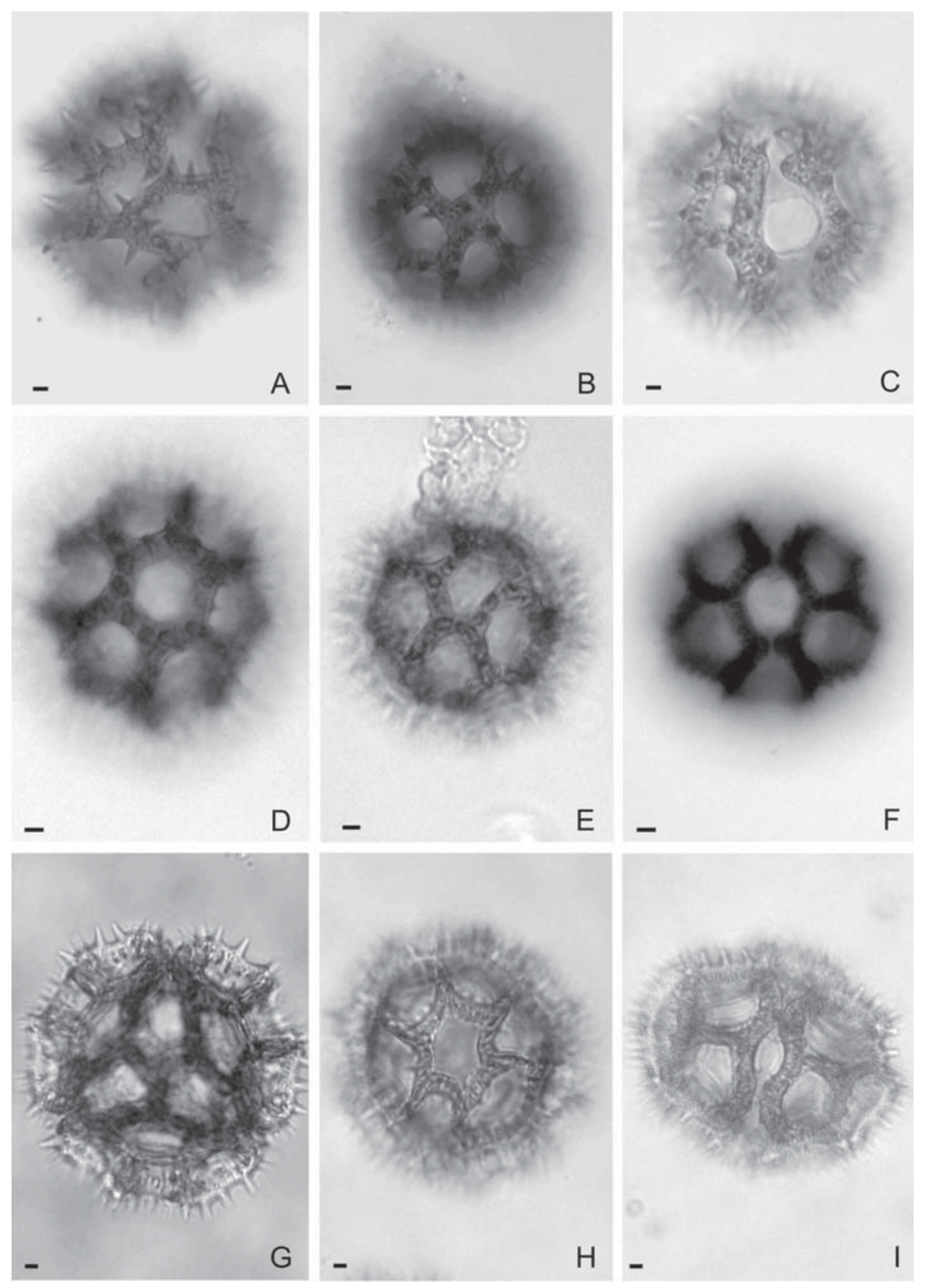

Figura 1. Granos de polen de Lepidaploa y Lessingianthus. A-C. Lepidaploa novarae, polen tipo "D”, A. vista polar, B. vista ecuatorial, mesocolpio, C. vista ecuatorial, apertura. D-F. Lepidaploa setososquamosa, polen tipo "C", D. vista polar, E. vista ecuatorial, mesocolpio, F. vista ecuatorial mostrando apertura. G-I. Lessingianthus hasslerianus, polen tipo "B”, G. vista polar, H. vista ecuatorial, mesocolpio, I. vista ecuatorial, poro. Escala $=10 \mu \mathrm{m}$.

Figure 1. Pollen grains of Lepidaploa and Lessingianthus. A-C. Lepidaploa novarae, pollen type "D", A. polar view, B. equatorial view, mesocolpium, C. equatorial view, aperture. D-F. Lepidaploa setososquamosa, pollen type "C", D. polar view, E. equatorial view, mesocolpium, F. equatorial view showing aperture. G-I. Lessingianthus hasslerianus, pollen type "B", G. polar view, H. equatorial view, mesocolpium, I. equatorial view, aperture. Scale $=10 \mu \mathrm{m}$. 

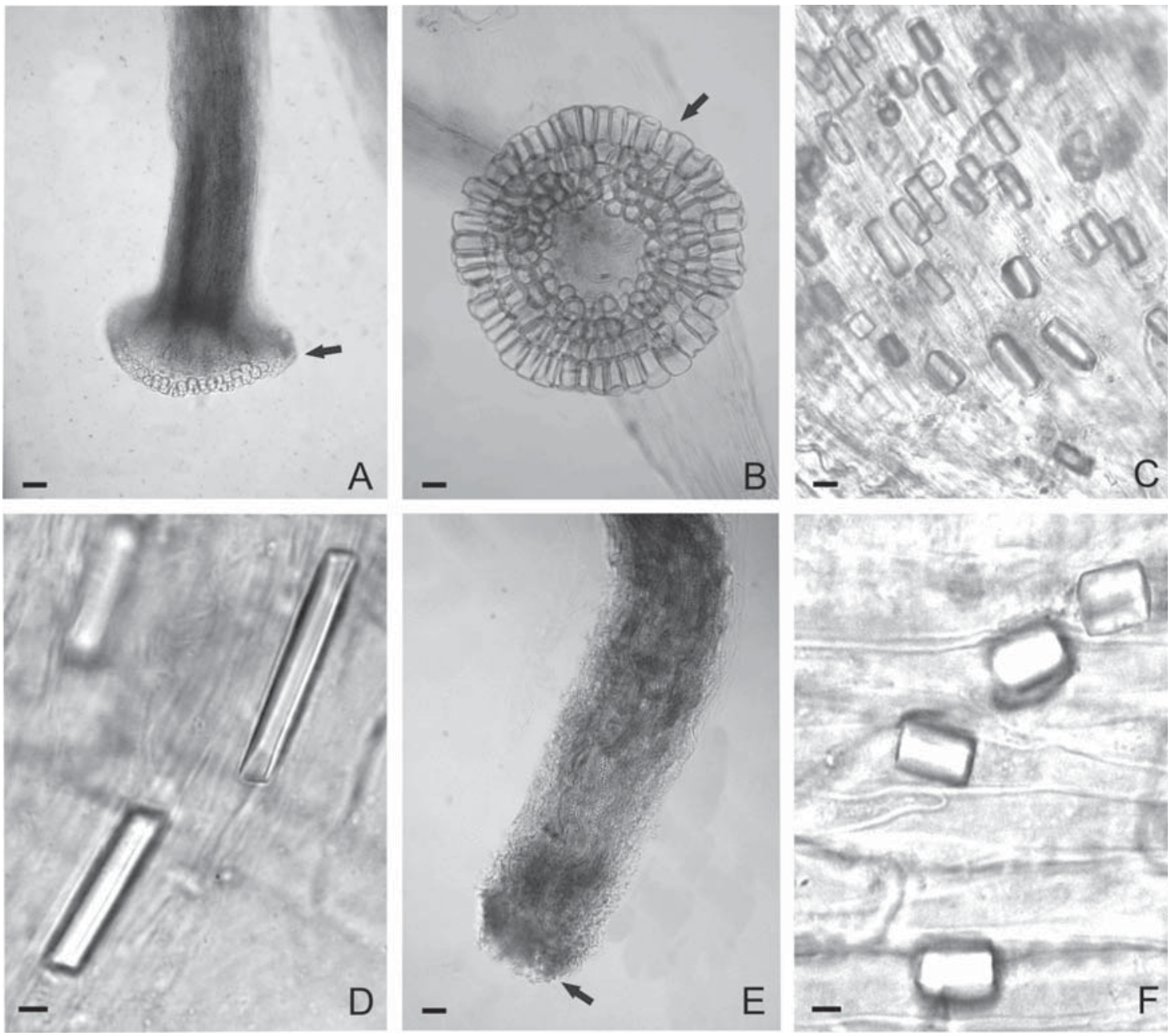

Figura 2. Microcaracteres florales de Lepidaploa y Lessingianthus. A-D. Lepidaploa novarae, A-B. base del estilo mostrando el nódulo basal (flecha), C-D. cristales prismáticos del fruto. E-F. Lessingianthus bellulus, E. base del estilo sin nódulo en la base (flecha), F. cristales cúbicos del fruto. Escala: A-B, E= $30 \mu \mathrm{m}, \mathrm{C}-\mathrm{D}, \mathrm{F}=10 \mu \mathrm{m}$.

FIGURE 2. Floral microcharacters of Lepidaploa and Lessingianthus. A-D. Lepidaploa novarae, A-B. style base showing basal node (arrow), C-D. prismatic crystals of the fruit. E-F. Lessingianthus bellulus, E. style base without node in the base (arrow), F. cubic crystals of the fruit. Scale: A-B, $E=30 \mu \mathrm{m}, \mathrm{C}-\mathrm{D}, \mathrm{F}=10 \mu \mathrm{m}$.

Los granos de polen de Lepidaploa novarae fueron estudiados por Dematteis \& Pire (2008), quienes encontraron que esta especie presenta granos de polen tipo "Aa", el cual es tricolporado, equinolofado con el tectum discontinuo, comprendiendo lofos que rodean lagunas irregulares; éste tipo de polen es semejante al tipo "A" presente en el género Vernonanthura $\mathrm{H}$. Rob. El polen tipo " $\mathrm{A}$ " es subequinolofado, con tectum continuo y lofos rodeando depresiones irregulares del tectum. Sin embargo, en el análisis realizado en este trabajo se observó que esta especie presenta en realidad polen de tipo "D", el cual no está presente en el género Vernonanthura, sino en Lepidaploa.
Además del tipo de polen, la presencia del nódulo en la base del estilo y de los cristales prismáticos en la pared del fruto indican que esta entidad pertenece al género Lepidaploa.

DISTRIBUCIÓN, HÁBITAT Y FENOLOGÍA: Esta especie se distribuye en el nordeste de Salta, en los Departamentos Santa Victoria y Orán. Vegeta en selvas a 1.400-1.800 m s.m. Florece entre septiembre y octubre.

Material adicional examinado: ARGEnTINA, Prov. Salta, Dpto. Orán, Aguas Blancas, Finca El Arrazayal, Río Pescado. 24-VI-1985. Palací 130 (CORD, LIL, MCNS). 
Lepidaploa setososquamosa (Hieron.) M.B. Angulo \& Dematt. comb. nov. Basónimo: Vernonia setososquamosa Hieron., Bot. Jahrb. Syst. 22: 684. 1897. TIPO: “Argentina. [Bolivia] Prov. Salta. [Dpto. Tarija] Nogalitos, cerca del río Yadasco, II.1873, Lorentz \& Hieronymus 1205” (lectotipo designado por Cristóbal \& Dematteis (2003: 47), CORD!).

Vernonia remotiflora (L.C.Ric.) var. aristifera Cabrera, Darwiniana 6: 326. 1944. TIPO: “Argentina. Prov. Salta, Dpto. Orán: Campo Grande, 23.XI.1927, Venturi 5534” (lectotipo designado por Cristóbal \& Dematteis (2003: 47), SI!, isotipos LIL!, US!).

Vernonia setososquamosa Hieron., descrita originariamente para la zona de Orán en la provincia de Salta (Argentina) fue incluida en varios tratamientos taxonómicos, pero en la mayoría de ellos se le ha asignado un estatus diferente. En la revisión de las Vernonieae argentinas, Cabrera (1944) la considera una variedad de $V$. remotiflora L. C. Rich. [=Lepidaploa remotiflora (L. C. Rich.) H. Rob.] sobre la base de las semejanzas morfológicas, tales como el tipo de inflorescencia, forma de la hoja, forma del involucro y cantidad de series de filarios. Este criterio fue seguido por estudios posteriores (Cabrera 1974, Cabrera \& Freire 1999). Por su parte, Robinson (1999), en un listado de las Vernonieae del continente Americano, incluye a esta entidad en la sinonimia de $V$. remotiflora. Una tercera propuesta es la de Cristóbal \& Dematteis (2003) quienes consideraron a Vernonia setososquamosa y $V$. remotiflora como especies diferentes, sobre la base de algunos caracteres morfológicos como la presencia de pelos en la corola, el tamaño de los capítulos y el hábito de las plantas. Recientemente, Angulo \& Dematteis (2010) hallaron que ambas entidades difieren además en el número de cromosomas y la morfología polínica. Los datos anteriores indican que Vernonia remotiflora y $V$. setososquamosa constituyen dos especies diferentes y no variedades de una misma especie. Esta última presenta todos los caracteres distintivos del género Lepidaploa, tales como granos de polen tipo "C", estilo basal anular, cristales prismáticos en la pared del fruto, número básico $\mathrm{x}=15$, por lo cual se hace necesaria una nueva combinación a este último género.

DistribuCiÓn, HÁBITAT Y FENOLOGÍA: Crece en el noroeste de Argentina, en la provincia de Salta, en los departamentos Orán y Rosario de la Frontera en campos arenosos, y en Rosario de Lerma en laderas y lomadas sobre el dique Las Lomitas a los 1.400 m s.m. También habita en el chaco paraguayo, en suelos arenosos de los Departamentos Boquerón y Alto Paraguay. En Bolivia habita en campos altos del Departamento Santa Cruz. Florece entre diciembre y marzo.
Material adicional examinado: ARGEnTINA, Prov. Salta, Dpto. Orán, a $3 \mathrm{~km}$ de Orán. 20-X-1971. Vallejos 8 (LIL); Ruta 50, 2 km al W de San Ramón de la Nueva Orán. 4-XII-2005. Arbo et al. 8993 (CTES); Dpto. Rosario de Lerma, Dique Las Lomitas. 9-II-1997. Novara 10877 (CTES, MCNS), Dpto. Rosario de la Frontera, Rosario de la Frontera. 28-I-1947. O’Donell 4731 (LIL); Rosario de la Frontera. 28-I-1947. O’Donell 4757 (LIL, S). BOLIVIA, Dpto. Santa Cruz, Prov. Chiquitos, ca. $50 \mathrm{~km}$ NW of Roboré. 6-II-1995. Abbott \& Mostacedo 16130 (USZ); Prov. Florida. 800-900 m. X-1910. Herzog 1279 (L); Buena Vista, 12-II1921, Steinbach 3286 (BAF, S); Los Aguaíces. 22-XI-1958. Meyer 22035 (LIL, LP); Prov. Warnes, Pampa de Viru-Viru, $17 \mathrm{~km}$ al $\mathrm{N}$ de la ciudad Santa Cruz, aeropuerto Internacional Viru Viru. 11-II-1994. Menacho \& Gutiérrez 438 b (LPB, USZ); Prov. Ibáñez, $1 / 2 \mathrm{~km}$ del Río Pejí, entre Mora y Santa Cruz de la Sierra. 30-III-2006. Dematteis et al. 1994 (CTES). PARAGUAY, Dpto. Alto Paraguay, Tyto. Gabino Mendoza, Gral. Garay. 31-III-1995. Degen \& Mereles 3298 (CTES, FCQ). Dpto. Boquerón, Nueva Asunción: km 695 de la Ruta Trans Chaco. 26-III-1986. Brunner 1677 (G); Ruta Transchaco, km 690. 7-V-1988. Charpin \& Ramella 21413 (G); Parque Valle Natural, 12 km S de Filadelfia. 28II-1991. Vanni et al. 2476 (CTES); Parque Valle Natural, 25 km al S de Filadelfia.7-XII-1992. Krapovickas \& Cristóbal 44197 (CTES, SI); 25 km al SE de Nueva Asunción. 15-V1994. Krapovickas et al. 45468 (CTES, SI).

\section{Lessingianthus}

El análisis de la morfología polínica y de algunos microcaracteres florales en seis especies ubicadas previamente en Vernonia permitieron transferir estos taxones al género Lessingianthus.

Todas las entidades analizadas presentaron granos de polen tipo "B" (Fig. 1 G-I), característico del género Lessingianthus. Estos fueron radialmente simétricos, oblatoesferoidales $(\mathrm{P} / \mathrm{E}=0,99)$, tricolporados, equinolofados. Los colpos son largos, con los ápices visibles en vista polar. El tamaño de los granos de polen es grande, $\mathrm{P}=45,7(67,2)$ $69,0 \mu \mathrm{m}, \mathrm{E}=45,6(67,1) 68,8 \mu \mathrm{m}$. El grosor de la exina, excluyendo las espinas, osciló entre 6,7 y 8,6 $\mu \mathrm{m}$. El tectum es discontinuo y comprende lagunas rodeadas por lofos, las lagunas están dispuestas en un patrón más o menos regular. La superficie del tectum es densamente microperforada, con espinas. Las espinas presentan una distribución linear a lo largo de los muros de los lofos, las cuales oscilaron en longitud entre 2,6 y $4,2 \mu \mathrm{m}$.

Todas las especies presentaron el estilo normal (Fig. 2 E), es decir, sin el nódulo en la base del mismo y los cristales cúbicos en la pared del fruto (Fig. 2 F). 
Lessingianthus bellulus (Dematt.) M.B. Angulo, comb. nov. Basónimo: Vernonia bellula Dematt., Candollea 55: 308. 2000. TIPO: "Paraguay. Dpto. Canendiyú: Entre Ype Hú y Capitán Bado, a 10 km de Itanará, 5.II.1982, Fernández Casas \& Molero 5989" (holótipo, MA, no visto; isotipos, CTES!, G!).

Lessingianthus bellulus está relacionada a L. ramellae (Cabrera) H. Rob. y L. pumillus (Vell.) H. Rob., de los cuales puede distinguirse por la forma y tamaño de las hojas. Lessingianthus bellulus tiene hojas ovadas-lanceoladas $\mathrm{u}$ oblongas-lanceoladas de $6-10 \mathrm{~cm}$ de long, mientras que L. pumillus tiene hojas lineares de $10-18 \mathrm{~cm}$ de long, y $L$. ramellae hojas lanceoladas de 2,5-4 $\mathrm{cm}$ de long.

Distribución, HÁBITAT Y FENOLOGÍA: Crece en campos altos, principalmente en cerrados del este de Paraguay, en el Departamento de Canindeyú y en el sur de Mato Grosso do Sul en Brasil. Esta especie era conocida solamente para el este de Paraguay, en el Departamento de Canindeyú (Cabrera \& Dematteis, 2009). En el presente trabajo se amplía el área de distribución conocida de dicha entidad, la cual es registrada por primera vez para el estado de Mato Grosso do Sul, Brasil. Florece entre diciembre y febrero.

Material adicional examinado: BRASIL, Mato Grosso do Sul, Rod. MS-295, Fazenda Santa Paula, 18 km O de Tacuru. 18-II-1993. Hatschbach et al. 58687 (CTES, MBM).

Lessingianthus centauropsideus (Hieron.) M.B. Angulo \& Dematt. comb. nov. Basónimo: Vernonia centauropsidea Hieron., Bot. Jahrb. Syst. 40: 353. 1908. TIPO: "Bolivia [Argentina]: in faucibus rivi prope [Los] Toldos haud procul a Bermejo, 1900 m, 26.XI.1903, Fiebrig 2258 [2248]" (holotipo, B; isotipo, G!).

Lessingianthus centauropsideus se encuentra relacionada a L. kuntzei (Hieron.) Dematt. de Bolivia y L. asteriflorus (Mart. ex DC.) H.Rob. de Paraguay y Brasil, de las cuales puede distinguirse por la inflorescencia más densa y los filarios internos glabros o con pelos solamente en el ápice. Además, L. centauropsideus presenta el haz de las hojas glabro o muy laxamente piloso y los filarios son escariosos, en tanto que L. asterifolius presenta el haz de la hoja lanoso y los filarios externos foliáceos. Por su parte, L. kuntzei se diferencia de las otras dos entidades por presentar los filarios foliáceos.

DisTRIBUCIÓN, HÁBITAT Y FENOLOGÍA: Especie conocida solamente para el departamento Santa Victoria en el norte de la provincia de Salta (Argentina) y el sur de Bolivia. Crece en lomadas y campos graminosos de altura. Si bien se trata de una especie con distribución relativamente restringida, es bastante abundante y forma grandes poblaciones, que resultan muy vistosas en la época de floración, en los meses de diciembre y enero.

Material adicional examinado: ARGENTINA, Prov. Salta, Dpto. Santa Victoria, camino de Los Toldos al Lipeo, a 7 km de Los Toldos. 11-XII-1973. Legname \& Cuezzo 9965 (LIL); camino de Los Toldos al Lipeo, a $\pm 15 \mathrm{~km}$ de Los Toldos. 11-XII-1973. Legname \& Cuezzo 9935 (LIL); camino de Los Toldos al Lipeo, a $\pm 4 \mathrm{~km}$ de Los Toldos. 9-XI-1975. Cuezzo et al. 12000 (LIL); Parque Nacional Baritú, camino a lo de Don Pablo Flores. 4-XII-1997. Hilgert 2031 (MCNS); ruta 40, $5 \mathrm{~km}$ de Los Toldos hacia Lipeo. 5-XII-2005. Arbo et al. 9107 (CTES). BOLIVIA, Dpto. Chuquisaca, Prov. Azurduy, 4-XII-1999, Wood 15293 (K); Prov. Tomina, sin indicar localidad, XII-1845/I-1846, Weddell 3845 (P). Dpto. Cochabamba, Salida de Epizana, carretera de Cochabamba. 20-II-1979. Ceballos et al. 411 (G).

Lessingianthus glabratus (Less.) H.Rob. var. angustifolius (Cabrera) Dematt. comb. nov. Basónimo: Vernonia glabrata Less. var. angustifolia Cabrera, Darwiniana 6: 317. 1944. TIPO: "Argentina, Prov. Misiones, Garupá, 19.II.1930, Rodríguez 85” (holotipo, LP!; isotipo, SI!).

Se diferencia de la variedad típica por las hojas linearlanceoladas, es decir mucho más angostas y largas, de 9-20 x 0,4-1,3 cm, y capítulos menores, con involucro de 9-10 x $12-15 \mathrm{~mm}$.

Distribución, HÁBITAT Y FENOLOGiÁ: Su área de distribución geográfica abarca el sur de Brasil, Paraguay oriental y el noreste Argentino. Se la encuentra en campos bajos en la proximidad de ríos, también como maleza en cultivos de arroz. Florece entre enero y abril.

Material adicional examinado: ARGEnTINA, Prov. Misiones, Dpto. Candelaria, Santa Ana. 15-I-1913. Rodríguez 723 (BA, CTES, SP); Dpto. San Ignacio, San Ignacio, ad fl. Yabebiry. 14-I-1908. Ekman 1161 (S). Prov. Corrientes, Dpto. Empedrado, Estancia Las Tres Marías, 19-IV-1962, Pedersen 6469 (C, CORD, P); Dpto. Itatí, Estancia Tuyutí. 21-IV-1960. Pedersen 5532 (C, CORD, CTES, P); Dpto. Mburucuyá, Estancia Santa María. 9-II1970. Pedersen 9639 (C, CTES, MBM, P).

Lessingianthus hasslerianus (Chodat) M.B. Angulo, comb. nov. Basónimo: Vernonia hassleriana Chodat, Bull. Herb. Boisser ser. 2, 2: 302. 1902. TIPO: "Paraguay. Dpto. Canindeyú: en campo San Blas (Yerutí), XII.1898/1899, Hassler 5774" (holotipo, G!).

Lessingianthus hasslerianus es afín a L. sellowii (Less.) H. Rob. de la cual puede diferenciarse fácilmente por la 
forma de las hojas; la primera presenta hojas lanceoladas o elípticas mientras que la última posee hojas obovadas u oblanceoladas.

DisTRIBUCIÓN, HÁBITAT Y FENOLOGÍA: Crece en campos serranos del este de Paraguay, desde el departamento de Amambay hasta el de Itapúa. Florece entre noviembre y enero.

Material adicional examinado: PARAGUAY, Dpto. Amambay, Sierra de Amambay, in campis siccis Punta Porá. XII-1907/1908. Rojas 9910 (BAF, G, W). Dpto. Itapúa, Capitán Meza. 18-XI-1945. Montes 7161 (SI).

Lessingianthus mollissimus (D.Don ex Hook. \& Arn.) H.Rob. var. microcephalus (Hieron.) Dematt., comb. nov. Basónimo: Vernonia mollissima D.Don ex Hook. \& Arn. var. microcephala Hieron., Bot. Jahrb. Syst. 22: 680. 1897. TIPO: "Argentina. Prov. Misiones, Entre San Carlos y Arroyo Pindapoi, 12.X.1886, Niederlein 1439"(holotipo, B, destruido; isotipo, BA!). Lectotipo, designado aquí BA!

Esta entidad se diferencia de la variedad típica por tener capítulos de menor tamaño, con involucro de 6-7 mm long. x 9-12 mm diám. y flores de $\pm 14 \mathrm{~mm}$ long.

El material original analizado por Hieronymus (1827) fue depositado en el herbario de Berlín, el cual fue destruido en la Segunda Guerra Mundial. Por lo tanto, se designó como lectotipo al espécimen de BA.

DisTRIBUCIÓN, HÁBITAT Y FENOLOGÍA: Esta variedad es propia de Córdoba en el centro de Argentina continental y el extremo noreste en la provincia de Misiones y norte de Corrientes, Argentina. Florece en primavera y verano.

Material adicional examinado: ARgentina, Prov. Córdoba, Dpto. Punilla, ruta provincial 14, al NE de Icho Cruz. 21-I-2004. Pozner \& Belgrano 350 (CTES). Prov. Corrientes, Dpto. Ituzaingó, ruta Nacional 12 y Ea. San Borgita. 20-X-1974. Carnevalli 3480 (CTES); ruta 12, 2 km antes del límite con Misiones. 11-IX-1978. Cristóbal \& Krapovickas 1799 (CTES); San Borgita, 1 km S de ruta 12, camino a San Carlos. 19-I-1993. Schinini 27563 (CTES). Dpto. Santo Tomé, $23 \mathrm{~km}$ E de Ruta Nac. 14, camino a Colonia Garabí. 3-XII-1970. Krapovickas et al. 16956 (CTES); 15 km de Gdor. Virasosro, camino a Garrucho. 5-II-1972. A. Krapovickas et al. 21002 (CTES); ruta 37, $5 \mathrm{~km}$ E de Gdor. Virasoro. 14-XI-1974. Schinini \& Carnevalli 10455 (CTES); 36 km de Santo Tomé, ruta Prov. 40. 2-II-1976. Krapovickas \& Cristóbal 29098 (CTES). Prov. Misiones, Dpto. Apóstoles, San José, Escuela Agrotécnica Pascual Gentilini. 10-II-1978. Cabrera \& Sáenz 29086 (CTES). Dpto. Caingúas, Puerto Rico. 12-XI-1948. Montes 3342 (CTES). Dpto. Candelaria: Bompland. 22-III-1910. Rodríguez \& Girola 88 (CTES). Dpto. Capital, Arroyo Zaimán. 25-XI-1994. Dematteis et al.
373 (CTES); Entrada a Ea. El Porvenir. 6-II-1996. Dematteis \& Solis Neffa 506 (CTES). Dpto. San Javier: $30 \mathrm{~km}$ de L. N. Alem, camino a San Javier, sobre ruta 4. 13-III-1969. Krapovickas et al. 15210 (CTES); 23 km SE de San Javier, entre Itacaruaré y Santa María. 22-I-1976. Krapovickas \& Cristóbal 28841 (CTES).

Lessingianthus valenzuelae (Chodat) M.B. Angulo, comb. nov. Basónimo: Vernonia valenzuelae Chodat, Bull. Herb. Boisser ser. 2, 3. 641.1903. TIPO: "Paraguay. In Valle fluminis Y-acá inter rupes pr. Valenzuela, I.1900, Hassler 6996 (holotipo, G!, isotipo, G)”.

Especie próxima a L. glabratus (Less.) H. Rob., de la cual puede distinguirse por la forma de las hojas, y de los filarios. Lessingianthus valenzuelae presenta hojas ovadas, los filarios externos son subulados en la parte superior y los internos son agudos y mucronados, en cuanto a L. glabratus tiene hojas lanceoladas u oblanceoladas y los filarios son exteriores son lanceolados acuminados, los interiores rojizos, linear-oblongos, obtusos y mucronulados en el ápice. Robinson (1988) considera a esta especie un sinónimo de L. niederleinii (Hieron.) H.Rob., de la cual se diferencia por el tamaño y el ápice de las hojas, tal como lo señalan Cabrera \& Dematteis (2009). Lessingianthus niederleinii posee hojas de 5-11 cm long., cortamente apiculadas en el ápice, mientras que L. valenzuelae tiene hojas de tan sólo 2,2-3 cm long., con el ápice agudo.

Distribución, hÁBitat y fenología: Crece en los cerros y suelos secos de los Departamentos paraguayos de Cordillera y Paraguarí. Florece entre enero y marzo.

Material adicional examinado: PARAGUAY, Dpto. Cordillera, Links vom Piribebuy und südlich von Tobatí, Serranía nordlich von tal des Y-aguaí-guazú. 04-II-1903. Fiebrig 859 (G, K); Piribebuy, Piraretá, 10-III-1989, Soria 3194 (FCQ, SI). Dpto. Paraguarí, Cordillera de Mbatabí, près de Paraguarí. III-1881. Balansa 3058 (BAF).

\section{AGRADECIMIENTOS}

Este trabajo se realizó mediante el apoyo financiero del Consejo Nacional de Investigaciones Científicas y Tecnológicas (CONICET) y la Secretaría General de Ciencia y Técnica de la Universidad Nacional del Nordeste (SGCyT-UNNE), Argentina.

\section{BIBLIOGRAFÍA}

Anderson, L.E. 1954. Hoyer's solution as a rapid mounting medium for bryophytes. Bryologist 57: 242-247. 
Angulo, M.B. \& M. Dematteis. 2010. Pollen morphology of the South American genus Lessingianthus (Vernonieae, Asteraceae) and its taxonomic implications. Grana 49(1): $12-25$.

Angulo, M.B. \& M. Dematteis. 2010. La identidad de Vernonia setososquamosa (Asteraceae, Vernonieae): evidencias cromosómicas y palinológicas. Darwiniana 48 (1): 17-24.

Angulo, M.B. \& M. Dematteis. 2012. Cytotaxonomy of some species of the South American genus Lessingianthus (Asteraceae, Vernonieae). Plant Systematics \& Evolution 298(2): 277-285.

Borges, R.A.X. \& M. Dematteis. 2008. A new species of Lessingianthus (Asteraceae: Vernonieae) from Minas Gerais, Brazil. Brittonia 60(4): 377-381.

Cabrera, A.L. 1944. Vernonieas Argentinas (Compositae). Darwiniana 6 (3): 265-379.

Cabrera, A.L. 1974. Compositae. En: Flora Ilustrada de Entre Ríos, vol. 6 (6) (ed. A. Burkart), pp. 106-554.

Cabrera, A.L. \& M. Dematteis. 2009. Compositae VI. Asteroideae. Tribu Vernonieae. En: L. Ramella \& P. Perret (eds.), Flora del Paraguay, vol. 39, pp. 65-268. Conservatoire et Jardin Botaniques de Geneve \& Missouri Botanical Garden, Geneve, Switzerland.

Cabrera, A.L. \& S.E. Freire. 1999. Asteraceae, Vernonia. En: F.O. Zuloaga \& O. Morrone (eds.), Catálogo de las Plantas Vasculares de la República Argentina, vol. II. Monographs in Systematic Botany from the Missouri Botanical Garden 74: 339-347.

Cristóbal, C.L. \& M. Dematteis. 2003. Asteraceae XVIII. Tribu I. Vernonieae. Flora Fanerogámica Argentina 83: 3-53.

Dematteis, M. 2006. Two new species of Lessingianthus (Vernonieae, Asteraceae) from the Brazilian highlands. Botanical Journal of the Linnean Society 150(4): 487493.

Dematteis, M. 2008. New species and new combinations in the South American genus Lessingianthus (Vernonieae, Asteraceae). Edinburgh Journal of Botany 65(3): 359368.

Dematteis, M. \& S.M. Pire. 2008. Pollen morphology of some species of Vernonia sensu lato (Vernonieae, Asteraceae) from Argentina and Paraguay. Grana 47(2): 117-129.

Dematteis, M. \& M.B. Angulo. 2010. Additions to the genus Lessingianthus (Vernonieae, Asteraceae) from South America. Rodriguésia 61(2): 233-241.

Dematteis, M. \& M.B. Angulo. 2012. New species of Lessingianthus (Asteraceae, Vernonieae) from Brazil and Paraguay. Blumea 57: 109-113.

Erdtman, G. 1966. Pollen morphology and plant taxonomy. Angiosperms. Hafner, New York.

JoNES, S.B. 1979. Synopsis and pollen morphology of Vernonia (Compositae: Vernonieae) in the New World. Rhodora 81: 425-447.

Keeley, S.C. \& S.B. Jones. 1979. Distribution of the pollen types in Vernonia (Vernonieae: Asteraceae). Systematics of Botany 4: 195-202.

Keeley, S.C. \& H. Robinson. 2009. Vernonieae. In: V.A. Funk, A. Susanna, T.F. Stuessy TF \& J. Bayer (eds.), Systematics, evolution and biogeography of Compositae, pp. 439-469. International Association for Plant Taxonomy, Vienna, Austria.

Punt, W., J.S. VAn Weenen \& W.A.P. Van Oostrum. 1994. Glossary of Pollen and Spore terminology. LPP Foundation, LPP Contributions series $\mathrm{N}^{\circ} 1$. The Netherlands: University of Utrecht.

Robinson, H. 1988. Studies in the Lepidaploa complex (Vernonieae: Asteraceae). IV. The new genus Lessingianthus. Proceedings of the Biological Society of Washington 101: 929-951.

RoBinson, H. 1990. Studies in the Lepidaploa complex (Vernonieae: Asteraceae). VII. The genus Lepidaploa. Proceedings of the Biology Society of Washington 103(2): 464-498.

Robinson, H. 1992. A new genus Vernonanthura (Vernonieae, Asteraceae). Phytologia 73(2): 65-76.

Robinson, H. 1999. Generic and subtribal classification of American Vernonieae. Smithsonian Contributions to Botany 89: 1-116.

Robinson, H. 2007. Tribe Vernonieae. In: J. Kadereit \& C. Jeffrey (eds.), The families and genera of vascular plants, vol 8. Asterales, pp. 165-192. Springer, Berlin, Germany. 